Part three tabulates to $3 \mathrm{D}$ that zero of $u \sin x-\cos x+e^{-u x}$ which lies between $\pi$ and $2 \pi$ for $u=.1(.01) .3(.02) 2$ and $\sqrt{u}=0(.02) .5$. The author states that the last figure should be correct to within 0.7 of a unit. Linear interpolation yields full accuracy and first differences are provided.

Midwest Research Institute YUDELL L. LUKE

Kansas City, Missouri

\title{
MATHEMATICAL TABLES-ERRATA
}

In this issue references have been made to errata in RMT 1022, 1032.

214.--E. P. Adams \& R. L. Hippisley, Tables of Elliptic Functions, Smithsonian Miscellaneous Collections, v. 74, no. 1, Washington 1939, 1947.

The heading of page 294

$$
\begin{aligned}
\text { for } E^{\prime} & =1.5629622295 \\
\text { read } E^{\prime} & =1.5631622295 .
\end{aligned}
$$

Box 342

\section{H. J. HAuer}

China Lake, California

215. - (1) A. A. Gershun, "Berechnung des Volumleuchtens," Physikalische Z. d. Sowjetunion, v. 2, 1932, p. 149-185 [MTAC, v. 2, p. 191].

(2) E. SchmidT, "Die Berechnung der Strahlung von Gasräumen," Zs. Verein Deutscher Ingenieure, v. 77, 1933, p. 1162-1164.

(3) S. Goldstein, "On the vortex theory of screw propellers," Roy. Soc., London, Proc., v. 123A, 1929, p. 440-465.

Three tables are given in (1), on p. 172, 175, and 180, respectively.

Table I, containing the function

$$
F_{1}(x)=1-(1-x) e^{-x}+x^{2} \operatorname{Ei}(-x)=1-2 x^{2} \int_{x}^{\infty} \frac{e^{-t}}{t^{3}} d t
$$

to $4 \mathrm{D}$ for $x=0(.01) .02, .05, .1, .2(.2) 1,1.6(.4) 2.4,3,4$, was read against the same function given in (2) in complementary form on p. 1163, also to $4 \mathrm{D}$ mainly, for $x=0(.01) .02, .05, .1(.1) 1.0(.2) 2.0,2.4,2.5,3(1) 5$. The discrepancies, and the extra values given in (2), were checked, revealing the following errors in (1) and (2):

$\begin{array}{cccc}(1) & F_{1} & \text { for } & \text { read } \\ & 0.4 & .4925 & .4854 \\ & 3.0 & .9822 & .9821 \\ & & & \\ \text { (2) } & 0.3 & .6000 & .6001 \\ 0.9 & .2516 & .2514 \\ 1.2 & .1680 & .1679 \\ 1.4 & .1296 & .1292 \\ 1.6 & .1011 & .0998 \\ 1.8 & .0777 & .0774 \\ 2.5 & .0328 & .0326 \\ 4.0 & .00545 & .00552 \\ 5.0 & .00175 & .00176\end{array}$


Table II, of the function

$$
F_{2}(x)=2 \sum_{1}^{\infty} \frac{(-1)^{p+1} x^{p}}{(p+2) p !}=1-\frac{2}{x^{2}}\left(1-(1+x) e^{-x}\right)
$$

was recomputed. The following values are in error:

$\begin{array}{ccc}F_{2} & \text { for } & \text { read } \\ 2.8 & .8039 & .8038 \\ 4.0 & .8863 & .8864 \\ 5.0 & .9289 & .9232\end{array}$

Table III gives $F_{x}=1-\int_{0}^{\pi / 2} e^{-x \cos t} \cos t d t=\frac{1}{2} \pi\left[I_{1}(x)-L_{1}(x)\right]$, where $I_{1}$ and $L_{1}$ are Bessel and Struve functions of imaginary argument. Comparison with (3) indicated one error in (1)

$$
x=4 \quad \text { for } 9281 \quad \text { read } 9271
$$

and two in (3)

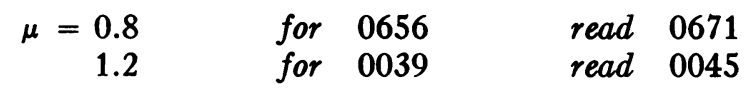

Scientific Computing Service, Ltd.

$$
\text { DAPHNE P. KILNER }
$$

London, England

216.-P. R. E. Jahnke \& F. EMde, Tables of Higher Functions. 4th (revised) ed. Leipzig, 1948.

This edition gives on p. 9 a more extensive table of the maxima and minima of the sine integral, that is, si $(x)$ for $x=\pi(\pi) 24 \pi$. Comparing these values with those obtained from interpolating in the NBS tables we find the seven errata:

$\begin{array}{cc}\pi^{-1} x & \text { for } \\ 18 & -0.007673 \\ 19 & +0.006744 \\ 20 & -0.005907 \\ 21 & +0.005151 \\ 22 & -0.004463 \\ 23 & +0.003834 \\ 24 & -0.008258\end{array}$

Moore School of Electrical Engineering

University of Pennsylvania

Philadelphia, Penn.

$$
\begin{gathered}
\text { read } \\
-0.017673 \\
+0.016744 \\
-0.015907 \\
+0.015151 \\
-0.014463 \\
+0.013834 \\
-0.013258
\end{gathered}
$$

H. J. GRAY, JR.

On p. 239 the entry immediately to the right of 6.3 in the $x$ column should be shifted down one line. Thus $-H_{1}^{(1)}(i 6.30)=0.0^{3} 6170$. The first significant figure of the function does not change from 6 to 5 until $x$ is between 6.32 and 6.33 . 
217.-Nils Pipping, "Die Goldbachsche Vermutung und der GoldbachVinogradowsche Satz," Åbo, Finland, Akad., Acta Math. Phys., v. 11, no. 4, 1938, p. 1-25.

$\begin{array}{ccc}x & \text { for } m_{x} & \text { read } \\ 6944 & 61 & 37 \\ 10006 & 149 & 83 \\ 23926 & 47 & 17 \\ 31004 & 73 & 67\end{array}$

515 West 110th Street

New York 25

\section{UNPUBLISHED MATHEMATICAL TABLES}

In this issue there is a reference to an unpublished table in RMT 1041.

150[F].-D. D. WALL, Table of Wilson's Quotient. 11 leaves tabulated from punched cards. Deposited in the UMT FILE.

For each of the 709 primes $p \leq 5381$ the table gives the least positive remainder on division of $\{(p-1) !+1\} / p$ by $p$. This remainder is zero for $p=5,13$, and 563. The table was produced on the IBM Card Programmed Calculator. [See also $M T A C$, v. 5, p. 81, MTE 182.]

IBM Corporation

D. D. WALL

Los Angeles, California

\section{AUTOMATIC COMPUTING MACHINERY}

Edited by the Staff of the Machine Development Laboratory of the National Bureau of Standards. Correspondence regarding the Section should be directed to Dr. E. W. Cannon, 415 South Building, National Bureau of Standards, Washington 25, D. C.

\section{Discussions}

\section{ASYNCHRONOUS SIGNALS IN DIGITAL COMPUTERS}

It is frequently necessary, during the operation of a digital computer, to inject signals from sources that are not synchronized with the computer itself, for example, the manual signals. This operation may be initiated by pressing an appropriate push button. In this discussion, we will not be concerned with such problems as "bounce" of contacts, wavering pressure or the possibility of repeated operation because of completion of computation before the button is released, but only with the fact that the contact is made (or broken) at a random moment with respect to the computer timing pulses or "clock." Probably the most important source of automatically generated signals asynchronous with the computer proper is the input equipment. Whether data are introduced by magnetic tape, punched cards, manual keyboard or other means, it is generally introduced at a much lower rate than transfers within the computer itself and at intervals which do not synchronize with the main "clock." 